\title{
Die Bedeutung der Ausgangsfrage für die Bearbeitung des Theodizeeproblems ${ }^{1}$
}

\author{
Alexander Dietz \\ Theologische Fakultät der Universität Heidelberg, \\ Kisselgasse 1, D-69117 Heidelberg, alexander.dietz@wts.uni-heidelberg.de
}

\section{Einleitung}

Der Theodizee-Diskurs ist zweifelsohne ein "Dauerbrenner « in der Theologie und phasenweise auch in der Philosophie. Nach wie vor garantiert eine Lehrveranstaltung zu diesem Thema volle Seminarräume. Nach wie vor erscheinen dazu regelmäßig neue wissenschaftliche Veröffentlichungen. Das Theodizeeproblem ist ein Sammelbegriff für sehr unterschiedliche Fragen und Artikulationen im Blick auf die Spannung von Leidenserfahrung und Gottesglaube. Der Begriff »Theodizee« wurde von Leibniz kreiert und spezifisch gefüllt als Rechtfertigung Gottes vor dem Gerichtshof der Vernunft. ${ }^{2}$ Manche Wissenschaftler schlagen darum vor, den TheodizeeBegriff nur für Ansätze der Aufklärungszeit, die dezidiert in dieser Tradition stehen, zu verwenden und angesichts früherer sowie späterer Ansätze von theodizeeanalogen Fragen zu sprechen. ${ }^{3}$ Tatsächlich stellt die aufklärerische Form des Theodizeeproblems einen spezifischen Zugang dar. Gleichwohl legen nicht nur der faktisch verbreitete heutige Sprachgebrauch, sondern auch zeitübergreifende Berührungspunkte der Zugänge aufgrund vergleichbarer Erfahrungen und Vernunftbedürfnisse sowie aufgrund der Suche nach Antworten vor dem Hintergrund gemeinsamer philosophischer und biblischer Traditionen eine weite Verwendung des Theodizeebegriffs nahe.

Es wird darüber diskutiert, ob die Theodizeefrage eine sinnvolle Frage darstelle oder ein unwissenschaftliches Scheinproblem, ob es auf die Theodizeefrage eine Antwort gebe beziehungsweise geben könne oder nicht, ob das Stellen der Theodizeefrage dem Glauben widerspreche und vor allem natürlich, welche Antwortansätze anderen vorzuziehen seien und warum. Erstaunlicherweise wird dabei äußerst selten thematisiert, in welcher Form genau die Theodizeefrage, auf die man sich bezieht, jeweils vorausgesetzt

1 Die folgenden Ausführungen wurden im Rahmen meines Habilitationsverfahrens als Habilitationsvortrag vor der Theologischen Fakultät der Universität Heidelberg im Wintersemester 2010/11 vorgetragen.

2 Vgl. Gottfried Wilhelm Leibniz, Die Theodizee von der Güte Gottes, der Freiheit des Menschen und dem Ursprung des Bösen (1710), in: DeR s., Philosophische Schriften, Bd. II/1 und 2, Darmstadt 1985.

3 Vgl. Willi Oelmüller, Vorwort, in: Ders. (Hg.), Theodizee - Gott vor Gericht?, München 1990, 7-8, 7 . 
wird. Vielmehr wird in der Regel in der Einleitung eines Werkes zur Theodizee mittels eines altbekannten Büchner-, Dostojewski- oder Camus-Zitats an die emotionale Zustimmung des Lesers zur bleibenden Aktualität des Theodizeeproblems appelliert. Mitunter wird auch die Vielfalt der Formen, in denen das Problem im Laufe der Geschichte artikuliert wurde, kurz erwähnt, um anschließend jedoch unvermittelt in die inhaltliche Diskussion über Antwortansätze zur jeweils zugrunde gelegten Theodizeefrage einzusteigen, die bestenfalls am Rande erwähnt wird. ${ }^{4}$ Jeder Autor scheint dabei seine Formulierung der Theodizeefrage selbstverständlich als angemessene Grundformulierung der Theodizeefrage vorauszusetzen, anscheinend meist ohne sich bewusst zu machen, dass eben diese Grundformulierung sich von Autor zu Autor - zum Teil grundlegend - unterscheidet. Wenn man jedoch ernst nimmt, dass »das Theodizeeproblem « einen Sammelbegriff für sehr unterschiedliche Fragen darstellt, setzt eine sinnvolle Bearbeitung des Theodizeeproblems eine bewusste Benennung der genauen Ausgangsfrage und idealerweise eine sachliche Begründung für die Wahl eben dieser Frage voraus.

Jeder Seelsorger wird auf die Frage »Wie kann ich Gott noch vertrauen, nachdem er dieses Übel zugelassen hat? " anders antworten als auf die Frage »Besteht ein zwingender logischer Widerspruch zwischen den Annahmen, dass es Übel in der Welt gibt und dass ein allmächtiger, allweiser und allgütiger Gott existiert? «. Es handelt sich hier nicht um die gleiche Frage in lediglich unterschiedlicher Formulierung. Von der Wahl der Frage (und dem Fragekontext) hängt es ab, ob die Frage sinnvoll ist, ob sie dem Glauben widerspricht, ob es darauf eine befriedigende Antwort gibt und welche Antwort darauf die beste ist. Daraus folgt, dass der angemessene Umgang mit dem Theodizeeproblem maßgeblich von der Formulierung der angemessenen Ausgangsfrage (und einer präzisen Analyse des Fragekontextes) abhängt. Die Frageformulierung hängt zum Beispiel davon ab, ob die Frage im Horizont des Glaubens gestellt wird oder nicht, in existenzieller oder abstrahierender Perspektive, mit Gott als Adressat oder nicht, an welchem Topos die Frage dogmatisch verortet oder ob ein traditionell-theistischer Gottesbegriff zugrunde gelegt wird. Ingolf U. Dalferth weist am Beispiel der Geschichte des Theodizeeproblems nach, dass sogar dieselben Sätze Verschiedenes bedeuten können, wenn sie vor dem Hintergrund unterschiedlicher Fragekontexte geäußert werden. ${ }^{5}$ Ich behaupte selbstverständ-

4 Positive Ausnahmen in der neueren theologischen Literatur: Wilfried HÄRLE, Dogmatik, Berlin u.a. ${ }^{3} 2007$, 439ff., sowie Ingolf U. D A L FER TH, Leiden und Böses, Leipzig 2006, $172 \mathrm{ff}$.

5 So ging es beispielsweise Epikur bei seiner berühmten aporetischen Aussagenreihe zum Verhältnis von Gott und Übel nicht um eine Infragestellung der Existenz Gottes beziehungsweise der Götter, sondern um eine Kritik an der traditionellen griechischen Vorstellung eines wohlgeordneten Kosmos. Vgl. Ingolf U. D a Lfer th, Malum. Theologische Hermeneutik des Bösen, Tübingen 2008, $38 \mathrm{ff}$. 
lich nicht, dass es eine richtige Frage gebe, sondern ich vertrete die These, dass es Gruppen von Fragen gibt, die dem Glauben angemessener sind als andere, und Gruppen von Fragen, die theologisch mehr Potenzial haben als andere, und dass es sinnvoll ist, sich stärker als bisher im Theodizee-Diskurs mit der Formulierung der jeweiligen Ausgangsfrage zu beschäftigen, im Bewusstsein darum, dass von dieser Frage maßgeblich abhängt, was von der weiteren Problembearbeitung jeweils erwartet werden kann und was nicht.

\section{Ausgangsfragen in religionsphilosophischer Perspektive}

Es ist möglich, das Theodizeeproblem im Horizont des Glaubens (also aus einer Innenperspektive) oder nicht im Horizont des Glaubens (also aus einer Außenperspektive) zu bearbeiten. Es ist nicht so, dass grundsätzlich nur die Bearbeitung im Horizont des Glaubens eine Berechtigung hätte. Einzelne Fragestellungen aus einer Außenperspektive können vielmehr eine sinnvolle Ergänzung zur Auseinandersetzung mit dem Problem aus einer Innenperspektive darstellen. Im Blick auf beide Zugangsweisen lässt sich jeweils eine weitere Unterscheidung treffen, und zwar diejenige zwischen einer existenziellen Perspektive und einer abstrabierenden Perspektive. Die abstrahierende und nicht im Horizont des Glaubens verortete Perspektive auf das Theodizeeproblem kann als religionsphilosophische Perspektive bestimmt werden. Hier lassen sich wiederum zwei klassische Zugänge zum Problem benennen, die zu unterschiedlichen Ausgangsfragen führen, nämlich der logische und der empirische Zugang.

Ausgehend vom logischen Zugang lautet die allgemeine Ausgangsfrage für die Bearbeitung des Theodizeeproblems: »Besteht ein logischer Widerspruch zwischen den Annahmen, dass es Übel in der Welt gibt und dass ein allmächtiger, allweiser und allgütiger Gott existiert? "Varianten dieser Frage lauten: »Besteht ein logischer Widerspruch zwischen den Annahmen, dass es bestimmte Arten und Mengen von Übeln in der Welt gibt und dass ein allmächtiger, allweiser und allgütiger Gott existiert? « und »Besteht ein logischer Widerspruch zwischen den Annahmen, dass es Übel in der Welt gibt und dass ein allmächtiger, allweiser und allgütiger Gott diese Welt geschaffen hat? "Nicht selten wird die logische Inkonsistenz dieser Annahmen leichtfertig als evident behauptet und für die weitere Behandlung des Theodizeeproblems vorausgesetzt, deren Weg dann vorgezeichnet ist als Entscheidung, ob man die Annahme der Existenz, der Allmacht oder der Güte Gottes fallen lassen möchte.

Möglicherweise könnte diese Entscheidung ja auch aus anderen Gründen geboten sein, jedoch nicht aus logischen. Logisch inkonsistent sind zwei Annahmen nur dann, wenn es aus logischen Gründen keine mögliche Welt gibt, in der beide zugleich zutreffen können. Zur Widerlegung der Behauptung eines logischen Widerspruchs genügt es, einen möglichen Fall 
zu nennen, in dem beide Annahmen zugleich zutreffen könnten, unabhängig davon, ob es diesen Fall gibt oder wie unwahrscheinlich er auch sein mag. Ein solcher Fall lässt sich meines Erachtens leicht benennen mit der Annahme, dass es denkbar (d.h. logisch möglich) ist, dass Gott hinreichende Gründe dafür hat, nicht jedes Übel zu verhindern, auch wenn wir diese Gründe nicht kennen. Beispielsweise ist es denkbar, dass die Zulassung von Übeln in logisch notwendiger Weise (also nicht nur in faktischer, kausaler Weise) mit der Zulassung größerer Güter (zum Beispiel der menschlichen Freiheit) oder der Abwesenheit größerer Übel (zum Beispiel der Abwesenheit von Glücksempfinden) verknüpft ist. Oder in einer Variante formuliert: Wenn man annimmt, dass die Welt von einem allmächtigen, allweisen und allgütigen Gott erschaffen wurde, muss man - wenn man nicht in einen Widerspruch geraten will - annehmen, dass die Welt in unübertrefflicher Weise gut ist und die Übel also konstitutive Bestandteile ihres Gutseins sind und Gott damit einen hinreichenden Grund hat, sie zuzulassen. $^{6}$

Wohl gemerkt: Diese Argumentation besagt nichts im Blick auf die Frage, ob es einen Gott gibt und ob die Welt seine Schöpfung ist oder wie wahrscheinlich das ist. Denker wie Leibniz oder in neuerer Zeit Friedrich Hermanni beweisen mit diesen Argumenten nicht mehr und nicht weniger, als dass die Annahmen, dass es Übel in der Welt gibt und dass ein allmächtiger, allweiser und allgütiger Gott existiert, nicht logisch inkonsistent sind. Das ist wenig hilfreich für den angefochtenen Glauben und trägt nicht viel aus für die inhaltliche Diskussion anderer religionsphilosophischer oder theologischer Ausgangsfragen zum Theodizeeproblem. Aber andererseits ist es wahrscheinlich die einzige der möglichen Ausgangsfragen, auf die es immerhin eine eindeutige Antwort gibt. Ganz anders sieht es beim empirischen Problemzugang aus. Darum ist es wichtig, diese beiden Zugänge nicht, wie es häufig geschieht, zu vermischen, sondern sie zu unterscheiden.

Den meisten Religionsphilosophen reicht die Lösung des logisch-formalen Widerspruch-Problems nicht aus, sondern sie fragen, welche Argumente die überzeugenderen sind: die Argumente, die gegen die Annahme eines allmächtigen, allweisen und allgütigen Gottes angesichts des Übels in der Welt vorgebracht werden, oder deren Gegenargumente? Ausgehend vom empirischen Zugang lautet die allgemeine Ausgangsfrage für die Bearbeitung des Theodizeeproblems: »Lassen sich plausible Erfahrungs-Gründe für die Wahrscheinlichkeit der Annahme finden, dass unsere Welt trotz des Übels die Schöpfung eines allmächtigen, allweisen und allgütigen Gottes ist? " Varianten dieser Frage lauten: "Lassen sich die Annahmen der Existenz, Weisheit und Güte Gottes vor dem Gerichtshof der Vernunft angesichts des Übels in der Welt rechtfertigen? « und »Kann die sich aus dem $\mathrm{Zu}$ -

${ }^{6}$ Vgl. Friedrich Hermanni, Das Böse und die Theodizee - Eine philosophisch-theologische Grundlegung, Gütersloh 2002, 261ff. 
stand der Welt aufdrängende Vermutung der Nicht-Existenz oder Gleichgültigkeit Gottes widerlegt werden? «

Besonders forensische Formulierungen der Ausgangsfrage sind häufig erläuterungsbedürftig: Wer genau soll wen vor wem verteidigen beziehungsweise rechtfertigen? Ist der Angeklagte beziehungsweise der zu rechtfertigende Gott selbst oder eine bestimmte Eigenschaft Gottes, ein bestimmter Gottesbegriff, der Glaube, die Vernunft, die Wirklichkeit, ein bestimmtes Wirklichkeitsverständnis oder der Mensch? In welchen Fällen kann die Vernunft sich als Ankläger, Verteidiger und Richter verstehen und in welchen nicht? In der Formulierung der Ausgangsfrage schlägt sich oft auch schon die Entscheidung darüber nieder, ob die Anklage oder die Verteidigung die Beweislast zu tragen hat. Von dieser Entscheidung hängt das Ergebnis der Auseinandersetzung mit dem Theodizeeproblem auf der Grundlage einer religionsphilosophisch-empirischen Ausgangsfrage maßgeblich ab.

Die klassischen Argumente der Verteidigung (also ontologische Depotenzierung des Übels oder seine Ästhetisierung, Moralisierung, Individualisierung, Pädagogisierung und Instrumentalisierung sowie seine Zurückführung auf notwendige geschöpfliche Begrenztheit) sind jeweils von sehr unterschiedlicher Überzeugungskraft, beziehen sich jeweils auf begrenzte Geltungsbereiche (Erklärung entweder des metaphysischen oder des physischen oder des moralischen Übels) und können - als Konsequenz des abstrahierend-distanzierten Ansatzes - bestenfalls rationale, kaum jedoch emotionale Akzeptanz hervorrufen, wodurch sie häufig auch dem Vorwurf ausgesetzt sind, das Leiden der Opfer nicht ernst zu nehmen. Die Anklage ist im Blick auf die Argumente der Verteidigung um keine Gegenargumente verlegen. Aus Kants Ausführungen in seiner Abhandlung "Über das Misslingen aller philosophischen Versuche in der Theodizee «7 lässt sich schlussfolgern, dass sowohl die Verteidigung als auch die Anklage im religionsphilosophisch-empirischen Theodizeeprozess dann notwendig zum Scheitern verurteilt sind, wenn sie die Beweislast tragen beziehungsweise mit Beweisanspruch auftreten, da die begrenzte menschliche Vernunft außerstande ist, von sich aus das Verhältnis zu erkennen, in welchem unsere empirische Welt zu der göttlichen Weisheit steht. Der Anspruch, das Verhältnis der Welt zum Willen Gottes von einem externen Standpunkt aus durchschauen zu können, kann nicht eingelöst werden. Zudem sind Fragen wie die, ob eine andere Welt, in der es keine Reifung oder keine Freiheit, dafür aber auch kein Leiden gäbe, besser wäre als die wirkliche Welt, grundsätzlich nicht empirisch beantwortbar. Erkenntnistheoretisch folgt daraus der aporetische Charakter der religionsphilosophisch-empirischen Ausgangsfrage(n).

Die Frage der Beweislastverteilung wird in der Literatur zur Theodizeefrage lediglich von Wilfried Härle ausführlicher reflektiert. In der ers-

7 Vgl. Immanuel KAnT, Über das Mißlingen aller philosophischen Versuche in der Theodizee, in: Werkausgabe, hg. von Wilhelm Weischedel, Band XI, Frankfurt am Main 1974, 103-124. 
ten Auflage seiner Dogmatik weist er die Beweislast der Anklage zu, da eine Schöpfungsthese, die keinen Beweisanspruch erhebe, auch keine Beweislast tragen müsse. Danach folgt aus dem offenen Prozessausgang ein (unbefriedigender) Freispruch. ${ }^{8}$ In der zweiten und dritten Auflage seiner Dogmatik weist er die Beweislast (mit leichter Einschränkung, aber grundsätzlich) der Verteidigung zu, da von der Schöpfungsthese als realitätsbezogener Hypothese erwartet werden könne, dass sie gute Gründe beibringe und Einwände entkräfte. ${ }^{9}$ Danach müsste aus dem konstatierten offenen Prozessausgang eigentlich eine (unbefriedigende) Verurteilung folgen (möglicherweise - um im Bild zu bleiben - mit einer Senkung des Strafmaßes aufgrund mildernder Umstände). ${ }^{10}$ Auch wenn Härle diese Konsequenz nicht explizit zieht, so führt ihn der unbefriedigende Prozessausgang doch dazu, die Auseinandersetzung mit dem Theodizeeproblem auf eine andere Ebene, nämlich die Ebene des Gefühls und der Klage zu verlagern. Man könnte dies im Sinne meines Anliegens als Hinweis auf die grundlegende Bedeutung der Ausgangsfrage interpretieren.

\section{Ausgangsfragen in existenzieller Perspektive nicht im Horizont des Glaubens}

Den Ausgangspunkt des Theodizeeproblems bildet die Spannung von Leidenserfahrung und Gottesglaube. Sowohl Leidenserfahrungen als auch die Glaubensfrage als grundlegende Vertrauens- und Sinnfrage betreffen den Menschen im Kern seiner Existenz, und dort nicht nur in seinem Denken, sondern vor allem auch in seinem Fühlen. Darum wird vielfach eine Bearbeitung des Problems, die von einer Ausgangsfrage in abstrahierender Perspektive ausgeht, als unangemessen empfunden. Die Fragen in existenzieller Perspektive unterscheiden sich grundlegend darin, ob sie im Horizont des Glaubens formuliert werden oder nicht. Versteht man Glauben als daseinsbestimmendes Vertrauen, das sich einem unverfügbaren Erschließungsgeschehen verdankt, ${ }^{11}$ kann sich ein Mensch seine existenzielle Perspektive nicht aussuchen. Bei Ausgangsfragen für die Bearbeitung des Theodizeeproblems in existenzieller Perspektive außerhalb des Horizonts des Glaubens kann unterschieden werden zwischen einem agnostischen Zugang, der zur Formulierung einer offenen Frage führt, und einem atheistischen Zugang, der zur Formulierung einer lediglich rhetorischen Frage führt.

8 Vgl. Härle (s.o. Anm. 4), 442.452.

9 Ebd.

10 Da Härle selbst betont, dass alle Theodizeeversuche, die einen Beweisanspruch erheben, notwendig scheitern (aaO., 447), müsste m.E. infolge der Neuverteilung der Beweislast (aaO., 442) die Aussage, dass die Verteidigung gelingen kann (aaO., 443) revidiert werden mit grundsätzlichen Auswirkungen auf die Anlage des gesamten Kapitels.

11 Vgl. Härle (s.o. Anm. 4), 56.96. 
Ausgehend vom agnostischen Zugang lautet die allgemeine Ausgangsfrage für die Bearbeitung des Theodizeeproblems: »Wie kann ich angesichts des Leids in der Welt an einen guten Gott glauben? "12 Varianten dieser Frage lauten: »Wie kann ich angesichts meiner Leidenserfahrungen an einen guten Gott glauben? « und »Wie kann ich angesichts des Leids in der Welt an einen Sinn glauben? « Hier wird die Theodizeefrage zur Frage nach dem Grund der eigenen Existenz und nach dem Wesen des Glaubens. Die angemessene Antwort auf diese Fragen wird je nach Situation wohl weniger in Argumenten liegen, die keinen Glauben wecken können, als in einem Handeln, das den anderen Liebe erfahren lässt (Schweigen ertragen, Mitleiden, Helfen), und ggf. im Zeugnis-ablegen von der eigenen Hoffnung, wie es in 1 Petr 3,15 heißt.

Ausgehend vom atheistischen Zugang lautet die allgemeine Ausgangsfrage für die Bearbeitung des Theodizeeproblems: »Wie könnte ich angesichts des Leids in der Welt an einen Gott glauben? «, die - als eine lediglich rhetorische Frage - nicht auf eine Antwort wartet. Eine Variante des Atheismus ist in diesem Zusammenhang das - für den Umgang mit dem Theodizeeproblem in der Moderne typische - Phänomen des Protestatheismus, von dem man sprechen kann, wenn das ungelöste Theodizeeproblem die Ursache einer Entscheidung zum Atheismus darstellt, weil beispielsweise jede Rechtfertigung einer Annahme der Existenz Gottes angesichts des Leids als inakzeptable Rechtfertigung des Leids empfunden wird. Der Spezialfall eines sich als christlich verstehenden Protestatheismus sollte zur Vermeidung von Missverständnissen eher als eine Kritik an einem spezifischen, vielleicht theistischen Gottesverständnis bezeichnet werden, die aus einer Solidarisierung mit Leidenden und aus einer Einsicht in die ethische Verantwortung des Menschen resultieren kann.

Mit der Annahme der Nichtexistenz Gottes stellt sich zwar nach wie vor die Frage nach dem Umgang mit Leidenserfahrungen, aber nicht mehr als Theodizeefrage. Die Frage stellt sich nun entweder in der nihilistischen Form: "Wie kann ich Leidenserfahrungen für mich vermeiden, ohne dass ich sie im Allgemeinen als Rätsel oder Skandal wahrnehmen würde? « oder in der humanistischen Form: "Wie kann ich zur Verhinderung oder Linderung von Leiden beitragen? «13 Das Verstummen der Theodizeefrage im ersten Fall um den Preis einer Normalisierung des Übels sowie des Verzichts einer Hoffnung auf eine Überwindung des Übels, bedeutet eine Infragestellung des Menschen als eines sittlichen Subjekts im traditionellen Sinne, da dieser sich nicht mehr auf dem Weg eines sittlichen Urteils von

12 Die klassische Formulierung dieser Frage stammt von Boëthius: »Si quidem deus [...] est, unde mala? «, allerdings fügte er hinzu: »Bona vere unde, si non est? « BOёTHıus, De Consolatione Philosophiae I, Prosa 4. Und Thomas spitzte diesen Gedanken noch zu: »Si malum est, deus est." Thomas von Aquin, Summa contra gentiles III, 71.

13 Eine Variante stellt die Frage dar: "Wie kann ich den Willen zum Leben beziehungsweise das Begehren als Ursache meines Leidens überwinden?« (Schopenhauer, Buddhismus). 
der gegebenen Welt distanzieren kann. ${ }^{14}$ Im zweiten Fall rückt der Mensch tendenziell an Gottes Stelle, in der er die Übel der Welt verantworten muss. Odo Marquard spricht im Blick darauf von einer Verwandlung der Theodizee in eine Anthropodizee, die den Menschen überfordere, da sie ihn unter einen gnadenlosen Rechtfertigungsdruck setze, der seine Angewiesenheit auf einen gnädigen Gott, an den er jedoch gerade nicht mehr glauben könne, augenscheinlich mache. ${ }^{15}$

\section{Ausgangsfragen in theologisch-wissenschaftlicher Perspektive}

Nach der Auseinandersetzung mit Ausgangsfragen für die Bearbeitung des Theodizeeproblems außerhalb des Glaubenshorizonts, beschäftige ich mich nun mit Ausgangsfragen innerhalb des Glaubenshorizonts, in dem die Spannung von Leidenserfahrung und Gottesglaube als Anfechtung des Glaubens wahrgenommen wird. Diese Anfechtung des Glaubens kann wiederum in abstrahierender, d.h. hier in theologisch-wissenschaftlicher Perspektive bearbeitet werden oder sich in existenzieller Klage Ausdruck verschaffen. Forderungen nach einem Verzicht auf eine theologisch-wissenschaftliche Bearbeitung des Theodizeeproblems als eines vermeintlich glaubenslosen, überheblichen oder zynischen Unterfangens übersehen, dass die Artikulation des Problems im Horizont des Glaubens gerade ein Ausdruck des Festhaltenwollens am Gottvertrauen, an der Gottesbeziehung trotz aller Anfechtung ist, dessen Grundlage die - für den Gläubigen identitätsstiftenden - Erfahrungen mit Gott in der Vergangenheit sowie die Selbstoffenbarung Gottes in Jesus Christus darstellen. Vor diesem Hintergrund könnte umgekehrt der Verzicht seitens der Theologie auf eine Auseinandersetzung mit dem Theodizeeproblem im Blick auf die Angefochtenen und Trostbedürftigen, wenn nicht zynisch, so doch gleichgültig wirken. ${ }^{16}$ Angesichts des offenbaren Gottes kann die Theologie sich mit dem Problem auseinandersetzen, angesichts des verborgenen Gottes wird sie jedoch auf den Anspruch einer vollständigen Lösung verzichten müssen.

Für die Klassifizierung möglicher Ausgangsfragen in theologisch-wissenschaftlicher Perspektive sind zum einen die thematische Verortung des Theodizeeproblems innerhalb der Dogmatik und zum anderen der jeweils zugrunde gelegte Gottesbegriff zentral. Die meisten dezidiert theologischen Ansätze lassen sich einem Topos der Trias von schöpfungstheologischem, kreuzestheologischem und eschatologischem Zugang schwerpunktmäßig

\footnotetext{
14 Vgl. Gerd Neuhaus, Frömmigkeit der Theologie. Zur Logik der offenen Theodizeefrage, Freiburg u.a. 2003, 24f.

15 Vgl. Odo Marquard, Abschied vom Prinzipiellen. Philosophische Studien, Stuttgart 1981, 49.

16 Vgl. Werner Thiede, Der gekreuzigte Sinn. Eine trinitarische Theodizee, Gütersloh 2007, 55.
} 
zuordnen. Was den jeweils zugrunde gelegten Gottesbegriff angeht, so haben sich viele namhafte Theologen des 20. Jahrhunderts - nicht nur, aber insbesondere auch im Kontext des Theodizeediskurses - gegen einen Gottesbegriff ausgesprochen, den sie mit den Begriffen metaphysisch, theistisch, philosophisch oder aristotelisch kennzeichneten, und stattdessen einen Gottesbegriff gefordert, für den Begriffe wie relational, biblisch, christlich oder trinitarisch verwendet werden. Der (in diesem Sinne der Begriffsverwendung!) traditionell-theistische Gottesbegriff steht für ein Verständnis von Gott als unbeweglicher, unveränderlicher, leidensunfähiger, selbstbezüglicher, abstrakt omnipotenter überweltlicher Person oder Wirklichkeit, die alles verursacht und kontrolliert. Der (in diesem Sinne der Begriffsverwendung!) nicht traditionell-theistische Gottesbegriff steht für ein Verständnis von Gott als lebendiger, leidensfähiger, prozessualer, sich selbst begrenzender und entäußernder Kraft oder Beziehungswirklichkeit der Liebe.

Ausgehend vom schöpfungstheologischen Zugang unter Zugrundelegung eines traditionell-theistischen Gottesbegriffs lautet die allgemeine Ausgangsfrage für die Bearbeitung des Theodizeeproblems: »Müsste die Welt, da doch Gott ihr Schöpfer ist, nicht anders aussehen? "Varianten dieser Frage lauten: »Warum lässt Gott das Leiden in seiner Schöpfung zu? « und »Ist Gott der Urheber des Übels? « Diese Ausgangsfragen führen zu einer Bearbeitung des Theodizeeproblems in Form einer Aufzählung von teils mehr, teils weniger überzeugenden Gründen, die Gott für die Zulassung des Übels in der Schöpfung haben könnte. Diese Gründe sind in der Regel weitgehend identisch mit denen, die vom religionsphilosophisch-empirischen Zugang her formuliert wurden, sie werden hier lediglich theologisch reformuliert unter Verwendung der Kategorien Geschöpflichkeit, Sünde und Heiligung. Die Ambivalenz der Antworten (insbesondere aufgrund der Verabsolutierung von Kontingentem) und der daraus folgende aporetische Charakter der Ausgangsfrage entsprechen ebenfalls dem Befund des religionsphilosophisch-empirischen Zugangs.

Ausgehend vom schöpfungstheologischen Zugang unter Zugrundelegung eines nicht traditionell-theistischen Gottesbegriffs lautet die allgemeine Ausgangsfrage für die Bearbeitung des Theodizeeproblems: »Warum nimmt sich Gott im Schöpfungsprozess selbst zurück? « Varianten dieser Frage lauten: »Kann Gott das Leiden in der Schöpfung verhindern? « und »Löst sich die Spannung von Leidenserfahrung und Gottesglaube in Anbetracht des Erleidens des Schöpfungsprozesses durch Gott auf? " Diese Ausgangsfragen setzen einen Schöpfungsbegriff voraus, der zum einen die Unvollkommenheit und Erlösungsbedürftigkeit der Schöpfung radikal ernst nimmt und zum anderen die kritischen Einwände gegen einen traditionell-theistischen Gottesbegriff schöpfungstheologisch weiterdenkt. Auch wenn sich das Theodizeeproblem dadurch zunächst verschärft, ist gegen physikotheologische oder neuromantische Schöpfungsbegriffe die Ambivalenz, die erschreckende Fremdheit der Schöpfung zu betonen, in der Leben nur auf Kosten von Leben existiert und eine von Grausamkeit und Zerstörung geprägte Schat- 
tenseite der Schöpfung ${ }^{17}$ eine natürliche Erkenntnis der Güte Gottes und damit eine vom Vorfindlichen ausgehende Theodizee unmöglich macht. Michael Welker spricht sich für einen Schöpfungsbegriff aus, der die Vorstellungen von Schöpfung als theistischer Naturproduktion und von Geschöpflichkeit als absoluter Abhängigkeit zugunsten der Vorstellungen von Schöpfung als »Aufbau und Erhalt von Interdependenzzusammenhängen zwischen natürlichen und kulturellen geschöpflichen Bereichen " ${ }^{18}$ sowie von einer am Schöpfungsvorgang beteiligten Eigenaktivität des Geschöpflichen überwindet.

Insbesondere prozesstheologische Ansätze, wie der von David R. Griffin, ${ }^{19}$ bearbeiten das Theodizeeproblem vor dem Hintergrund von Ausgangsfragen wie den genannten und gelangen zu interessanten neuen Perspektiven, die zumindest auf den ersten Blick einen Weg aus der Sackgasse $\mathrm{zu}$ weisen scheinen, in welche die traditionellen Theodizeeversuche geraten sind. Auf der Grundlage eines durch ein verändertes Schöpfungsverständnis ${ }^{20}$ modifizierten Allmachtsbegriffs wird bei Griffin das Wirken Gottes in einer dynamisch vorgestellten Welt als schöpferisch-überredende Liebe gefasst, die auf die Zustimmung und das Mithandeln der Geschöpfe, und zwar nicht nur der Menschen, angewiesen ist. ${ }^{21}$ Gott ist weniger Lenker als vielmehr Kooperationspartner der Welt sowie selbst eingebunden in den schmerzvollen, unabgeschlossenen Evolutionsprozess. So gibt es jedoch nur eine geringe Hoffnung, dass Übel und Leid in der Welt irgendwann einmal überwunden werden können. Dieses eschatologische Defizit wird meist als entscheidende Schwäche prozesstheologischer Theodizeen wahrgenommen. ${ }^{22}$

Ausgehend vom kreuzestheologischen Zugang unter Zugrundelegung eines traditionell-theistischen Gottesbegriffs lautet die allgemeine Ausgangsfrage für die Bearbeitung des Theodizeeproblems: »Warum oder wozu musste Jesus Christus leiden? « Varianten dieser Frage lauten: »Warum gehören Nachfolge und Leiden zusammen? « und »Löst sich die Spannung von Leidenserfahrung und Gottesglaube in Anbetracht der Rechtfertigung des Menschen durch Gott auf? "Solche Fragen richten den Blick des Theo-

17 Vgl. Karl B A R Th, Die Kirchliche Dogmatik, Bd. III/3, Zürich 1950, $334 f$.

18 Michael Welker, Schöpfung und Wirklichkeit, Neukirchen-Vluyn 1995, 29.

$19 \mathrm{Vgl}$. Barbara Luk os CheK, Das Theodizeeproblem in prozesstheologischer Perspektive, Berlin 2006.

20 Schöpfung aus dem Chaos statt Schöpfung aus dem Nichts.

21 Bei Griffin liegt dies in der Natur der Macht Gottes begründet im Unterschied zu den Gedanken der freiwilligen Selbstbegrenzung Gottes bei Eberhard Jüngel oder der freiwilligen Selbstentäußerung Gottes bei Hans Jonas. Vgl. Eberhard JÜNGEL, Gottes ursprüngliches Anfangen als schöpferische Selbstbegrenzung, in: DERs., Wertlose Wahrheit. Zur Identität und Relevanz des christlichen Glaubens, München 1990, 151-162. Vgl. Hans Jonas, Der Gottesbegriff nach Auschwitz. Eine jüdische Stimme, Frankfurt 1987.

22 Daneben wird geltend gemacht, dass der Gedanke eines Erleidens der Schöpfung durch Gott der Bibel eher fremd sei. 
logen weg von Spekulationen über die Macht und Herrlichkeit Gottes hin auf das Kreuz, wodurch christliche Theologie in den ihr angemessensten Horizont gelangt. Im leidenden Christus gibt sich Gott zu erkennen als ein Gott, dessen Wesen Liebe ist, als ein Gott, der Leiden zulässt und sich selbst hineinbegibt, um zu offenbaren, dass die Welt unter der Macht der Sünde steht, aber dennoch nicht von Gott im Stich gelassen wird. Vielmehr reißt Gott die Welt aus dem Leid-Schuld-Zusammenhang heraus durch das Versöhnungsgeschehen (als dessen Subjekt und nicht Objekt Gott gedacht werden muss), durch Sühne (die als Schaffung heiler Beziehungen und nicht als Vergeltung gedacht werden muss), durch das Opfer Jesu Christi (das als sacrificium im Blick auf die Menschwerdung und nicht als Viktimisierung am Kreuz gedacht werden muss). In den synoptischen Evangelien stehen die Ankündigung der Leiden Jesu und der Ruf in die Nachfolge als Bindung an die Person Jesu Christi, zu der es gehört, sein Kreuz auf sich zu nehmen, in einem engen Zusammenhang (Mt 16,21ff., Mk 8,31ff., Lk $9,22 \mathrm{ff}.)^{23}$

Die Transformation der Frage nach der Rechtfertigung Gottes durch den Menschen in die Frage nach der Rechtfertigung des Menschen durch Gott im Christusgeschehen, wie sie insbesondere Karl Barth vornimmt, impliziert die Einsichten, dass der angefochtene Glaube letztlich auf die Gnadenbotschaft angewiesen ist und dass die endgültige Antwort auf das Theodizeeproblem nur von Gott erwartet werden kann, weil erstens die Güte der Schöpfung nicht aus der Welterfahrung, sondern nur aus der Rechtfertigung der Schöpfung durch Gott erkannt werden kann und weil zweitens nur Gott die Sünde, verstanden als Unglaube beziehungsweise Verfehlung der Bundesbestimmung, endgültig überwinden kann. Nach Barth hat sich Gott selbst gerechtfertigt, obwohl er das nicht nötig hatte, indem er, dessen Gerechtigkeit niemals ernsthaft in Frage stehen kann, sich als gerecht erwiesen hat, indem er den Menschen und die ganze Schöpfung gerechtfertigt hat. ${ }^{24}$ Die Grenze dieses Ansatzes liegt - neben der Gefahr einer Verharmlosung des Bösen ${ }^{25}$ - in der (gerade für den Menschen, dessen Glaube aktuell angefochten ist) geringen Zustimmungsfähigkeit zu den Aussagen, dass das

23 Dietrich Bonhoeffer präzisiert das Kreuz der Nachfolge, ohne dass Gnade zur billigen Gnade werde, als täglichen Kampf gegen die Anfechtungen. Vgl. Dietrich Bonhoeffer, Nachfolge, Gütersloh 2002, 30.81.

24 Vgl. Karl Barth, Die Kirchliche Dogmatik, Bd. IV/1, Zürich 1953, 633. Die Auferweckung Jesu Christi als göttliches Urteil über das Kreuzesgeschehen ist die Rechtfertigung Gottes selbst, die Rechtfertigung Jesu Christi und die Rechtfertigung der sündigen Menschheit. Vgl. aaO., $340 f$.

25 Indem die Sünde als Nichtiges definiert wird und indem die grundlegende Unterscheidung zwischen der Sünde und der Schattenseite der Schöpfung als Teil der guten Schöpfung nicht konsequent durchgehalten wird, wodurch die Sünde eine positive Funktion erhält. Vgl. Yong Sung KIM, Theodizee als Problem der Philosophie und Theologie. Zur Frage nach dem Leiden und dem Bösen im Blick auf den allmächtigen und guten Gott, Münster 2002, 175. 
Theodizeeproblem durch Gott erledigt worden sei und dass der Mensch nicht Fragender zu sein, sondern sich als Gefragter zu verstehen habe.

Ausgehend vom kreuzestheologischen Zugang unter Zugrundelegung eines nicht traditionell-theistischen Gottesbegriffs lautet die allgemeine Ausgangsfrage für die Bearbeitung des Theodizeeproblems: »Löst sich die Spannung von Leidenserfahrung und Gottesglaube angesichts des Mitleidens Gottes auf? « Eine Variante dieser Frage lautet: »Kann im Leiden eine besonders intensive Gottesgemeinschaft erfahren werden? «Im Kreuz offenbart sich der Zusammenhang von Liebe auf der einen Seite und Leiden (vgl. 1Kor 13,7), Verwundbarkeit und Ohnmacht auf der anderen Seite. ${ }^{26}$ Jürgen Moltmann geht so weit, das Leiden und den Tod Jesu Christi vor dem Hintergrund seiner Kritik an einem apathischen Gottesbegriff - trinitarisch reflektiert - als Leiden und Tod in Gott zu deuten. ${ }^{27}$ Daraus folgt, dass menschliches Leiden Gott nicht fremd ist und nicht von Gott trennen kann, ja dass eine Erfahrung der Liebe Gottes mitten im eigenen Leiden im Sinne kreuzesmystischer Ansätze möglich erscheint. Der Gedanke vom Mitleiden Gottes kann dem angefochtenen Gläubigen im Einzelfall durchaus Trost spenden. Aber die von manchen geäußerte Auffassung, dass der mitleidende Gott die endgültige Antwort auf die Theodizeefrage darstelle, ${ }^{28}$ vermag nicht zu überzeugen. Wie es Johann Baptist Metz in seiner bekannten Kritik an einer Verdoppelung, Verewigung und heimlichen Ästhetisierung des Leidens ${ }^{29}$ treffend formulierte, führt die Verohnmächtigung Gottes zu einer Preisgabe der christlichen Hoffnung auf eine Erlösung der sündigen Welt durch Gott. Wenn der Allmachtsbegriff zwar im Sinne einer Behauptung abstrakter Omnipotenz und Allkausalität aufgegeben, aber im Sinne einer Hoffnung, dass Gott einmal alle Tränen abwischen wird, wie es in Apk 21,4 heißt, ${ }^{30}$ festgehalten werden soll, dann führt der Gedanke des Mitleidens Gottes beziehungsweise diese Variante der Theodizee-Ausgangsfrage nicht zu einer Auflösung, sondern vielmehr zu einer Verschärfung des Problems. Im Horizont der christlichen Hoffnung wird diese Aus-

26 Vgl. dazu Stefan TовцеR, Jesu Gottverlassenheit als Heilsereignis in der Spiritualität Chiara Lubichs. Ein Beitrag zur Überwindung der Sprachnot in der Soteriologie, Berlin u.a. 2003.

27 Nicht theopaschitisch als Tod Gottes. Vgl. Jürgen Moltmann, Der gekreuzigte Gott. Das Kreuz Christi als Grund und Kritik christlicher Theologie, München ${ }^{5} 1987,144.179 .192$. Ihm wurde vorgeworfen, dass die Aussage vom Leiden Gottes in einen Widerspruch zur biblischen und theologischen Tradition gerate, sobald sie sich nicht mehr auf Jesus (im Sinne der communicatio idiomatum), sondern auf das Wesen Gottes beziehe. In späteren Veröffentlichungen ergänzt Moltmann das Leiden des Sohnes und des Vaters noch durch das Leiden des Geistes (der der leidenden Schöpfung einwohnt). Vgl. Jürgen MoltmanN, Gott in der Schöpfung. Ökologische Schöpfungslehre, München 1985, 113.

28 Vgl. Walter Kasper, Der Gott Jesu Christi, Mainz 1982, 244.

29 Vgl. Johann Baptist Metz, Theologie und Theodizee, in: Oelmüller (s.o. Anm. 3), 103 118, 117.

30 Vgl. Wolfgang Schoвerth, Gottes Allmacht und das Leiden, in: Werner H. Ritter u.a., Der Allmächtige. Annäherungen an ein umstrittenes Gottesprädikat, Göttingen ²1997, 43-67, 66. 
gangsfrage transformiert in die Frage: »Wie lange noch? «, die schon der Psalmbeter angesichts von Leid und Lästerungen ausgerufen hat. ${ }^{31}$

Ausgehend vom eschatologischen Zugang unter Zugrundelegung eines traditionell-theistischen Gottesbegriffs lautet die allgemeine Ausgangsfrage für die Bearbeitung des Theodizeeproblems: »Warum kommt die Erlösung erst nach einer Leidensgeschichte, warum nicht gleich der neue Himmel und die neue Erde? « Varianten dieser Frage lauten: »Warum geht es vielen Unschuldigen schlecht und vielen Gottlosen gut? «, wie Kohelet es formuliert (vgl. Koh 8,14 und Ps 73,2-16), und »Warum offenbart sich Gott manchen Menschen so, dass sie glauben können und gerettet werden, und anderen nicht? " Diese Fragen stellen sich vor dem Hintergrund der zum christlichen Glauben gehörenden Hoffnung, dass Gottes Gerechtigkeit eschatologisch vollständig offenbar werden wird, wenn Gott als Erlöser die Welt vollendet, Gericht hält und das Böse endgültig überwindet. Der Grund dieser Hoffnung ist das Evangelium von Jesus Christus, insbesondere die Auferweckung des Gekreuzigten. Die Hoffnung hebt jedoch die Anfechtung für den Glauben, die sich aus der Spannung zwischen Leidenserfahrung und Gottesglaube in der gegenwärtigen unerlösten Welt notwendig ergibt, nicht auf. Sie erzeugt vielmehr die genannten Fragen, die aufgrund des Gehalts der Hoffnung zwar nicht unlösbar sind, aber die aufgrund ihres Charakters erst im Eschaton beantwortet werden können, dann, wenn sie eigentlich gegenstandslos geworden sein werden, wie es in Joh 16,23a heißt: "An dem Tag werdet ihr mich nichts fragen." Antwortversuche, die dies ausblenden, sind notwendig unbefriedigend. In der Regel werden sie dem Einwand, dass nach christlicher Hoffnung eine Welt ohne Leid, Schmerz, Tod und Tränen möglich ist, gerade nicht gerecht und nehmen häufig Opfer scheinbar leichtfertig in Kauf. ${ }^{32}$ Jürgen Ebach formuliert treffend: "Nicht, dass das Leiden sinnvoll oder zweckgerichtet war oder als solches sich erweisen möge, ist zu hoffen, sondern dass es zu einem Ende kommt. ${ }^{33}$ Die Spitze des Theodizeeproblems lässt sich nach Luther in der Frage formulieren, "wie Gott den verdammt, der aus seinen eigenen Kräften nichts anderes tun kann als zu sündigen und schuldig zu werden «. ${ }^{34}$ Luther ermutigt den Glaubenden, darauf zu vertrauen, dass auch diese Frage sich im Licht der Herrlichkeit als lösbar erweisen wird.

31 Vgl. zum Beispiel Ps 74,10 oder Ps 119,84.

32 Vgl. Gerd Neuhaus, Frömmigkeit der Theologie. Zur Logik der offenen Theodizeefrage, Freiburg u.a. 2003, 50.

33 Jürgen Евасн, "Ein Sturm vom Paradiese her«. Walter Benjamins Geschichtsphilosophie und die hebräische Bibel, in: Ders., Ursprung und Ziel. Erinnerte Zukunft und erhoffte Vergangenheit, Neukirchen-Vluyn 1986, 48-74, 70.

34 Martin Luther, De servo arbitrio, in: Lateinisch-Deutsche Studienausgabe, Bd. 1, hg. v. Wilfried Härle, Leipzig 2006, 219-661, 657. Zuvor fragt Luther ähnlich, »warum die einen vom Gesetz erreicht werden, die anderen nicht, so dass jene die angebotene Gnade annehmen und diese sie verachten «. AaO., 405. 
Ausgehend vom eschatologischen Zugang unter Zugrundelegung eines nicht traditionell-theistischen Gottesbegriffs lautet die allgemeine Ausgangsfrage für die Bearbeitung des Theodizeeproblems: "Wie können wir als Vertreter Gottes zur Verhinderung oder Linderung von Leiden beitragen? « Dorothee Sölle gehört zu den Theologinnen und Theologen, welche die kritischen Einwände gegen einen traditionell-theistischen Gottesbegriff eschatologisch und ethisch weiterdachten. Sie fragt, wie ein christliches Leben unter Verzicht auf die Vorstellung eines allmächtigen himmlischen Wesens aussehen könnte. Danach sei Gott abwesend und müsse vertreten werden. ${ }^{35}$ Er habe keine anderen Hände als die der Menschen, die seinen Willen tun. ${ }^{36}$ Hingabe an Gott bedeute dann Hingabe an den Mitmenschen, in dem Gott begegne. Die Übersetzung des Satzes, dass Gott jeden liebt, sei die weltverändernde Praxis. ${ }^{37}$ Die Reaktion des Christen, der sich in diesem Sinne als Mitarbeiter Gottes verstehe, auf die Spannung von Leidenserfahrung und Gottesglaube bestehe darin, sich an die Seite der Leidenden zu stellen, leidschaffende soziale Strukturen zu verändern und dadurch das Leiden schrittweise zurückzudrängen. ${ }^{38}$ Vor dem Hintergrund einer säkularisierten und politisierten Eschatologie verschiebt sich auf diese Weise der Fokus der Ausgangsfrage hin auf die menschlichen Möglichkeiten, letztlich auf die menschlichen Möglichkeiten der Selbsterlösung. Hier wird deutlich, dass Sölles Ansatz in seiner Radikalität zwar interessant, aber doch auch unbefriedigend ist.

\section{Ausgangsfragen in existenzieller Perspektive im Horizont des Glaubens}

Jede Behandlung des Theodizeeproblems aus einer abstrahierenden Perspektive stößt an die Grenze des sich empörenden Gefühls angesichts der unmittelbaren Betroffenheit durch die Erfahrung des Leidens. Dieses Gefühl kann durch eine theoretische Antwort nicht beruhigt werden. Darum kommt Wilfried Härle zu dem Schluss: »Die Eröffnung der Möglichkeit der Klage (die auf Erhörung hoffen kann) ist das theologische Kriterium des angemessenen Umgangs mit dem Theodizeeproblem. " ${ }^{39}$ Klage ist die Form, in der die Anfechtung des Glaubens aufgrund der Spannung zwischen Leidenserfahrung und Gottesglauben in existenzieller Perspektive artikuliert wird. Hier steht die emotionale, praktische Leidbewältigung und nicht die Lösung eines theoretischen Gedankenkonflikts im Vordergrund.

\footnotetext{
35 Vgl. Dorothee Sölle, Stellvertretung. Ein Kapitel Theologie nach dem »Tode Gottes«, Berlin ${ }^{4} 1967,178 \mathrm{ff}$.

36 Vgl. Dorothee Sölle, Leiden, Stuttgart 2002, 168.

37 Vgl. Dorothee Sölle, Politische Theologie, Stuttgart 1982, 111.

38 Vgl. SÖLle (s.o. Anm. 36), 200.

39 Vgl. Härle (s.o. Anm. 4), 453.
} 
Verschiedene Bedürfnisse des Fragenden führen also zu unterschiedlichen Frageformulierungen. Das Theodizeeproblem kann nun in seiner umfassenden Bedeutung als Problem der Einheit der eigenen Lebensgeschichte ${ }^{40}$ beziehungsweise als Problem der Ich-Identität ${ }^{41}$ wahrgenommen werden. Angesichts der großen Bedeutung der Klage in der biblischen Tradition stellt Gotthard Fuchs fest, dass die neuzeitliche christliche Frömmigkeitspraxis »eigentümlich klaglos " ${ }^{42}$ geworden sei. Die Klage kann verschiedene Formen annehmen, wie fragen, anklagen, seufzen oder fluchen. Sie kann sich auf das eigene Leiden oder solidarisch auf das Leiden anderer beziehen. Sie kann Gott direkt als Adressaten wählen oder nicht.

Ausgehend vom Zugang der Klage nicht mit Gott direkt als Adressat lautet die allgemeine Ausgangsfrage für die Bearbeitung des Theodizeeproblems: »Wie kann ich Gott noch vertrauen, nachdem er das zugelassen hat? « Varianten dieser Frage lauten: »Wie konnte Gott das bloß zulassen? « und »Warum muss gerade ich leiden? « Die Klage hält die Theodizeefrage offen, anstatt sie zu verdrängen, oder vor ihr in Form von blindem Fideismus, Atheismus oder völliger Resignation zu verstummen. Die Klage kann ein Ausdruck des Standhaltens in der Anfechtung und des Festhaltens an der Hoffnung auf eine Überwindung des Übels sein. Diese Bedeutung der Klage kommt selbst dann noch bis zu einem gewissen Grad zur Geltung, wenn der klagende Gläubige auf einen Adressaten verzichtet beziehungsweise seine Klage die Form eines Selbstgesprächs ohne die Erwartung einer Antwort annimmt. Wählt der Klagende einen Freund oder Seelsorger zum Adressaten, kann dieser, angesichts der Unangemessenheit theoretischer Antworten auf existenzielle Fragen, ${ }^{43}$ meist lediglich seine eigene Antwort- und Hilflosigkeit zugeben und aushalten, möglicherweise die Klage verstärken oder den Klagenden dazu ermutigen, Gott direkt als Adressaten zu wählen, wodurch sich eine neue, fruchtbarere Frageperspektive eröffnen kann.

Ausgehend vom Zugang der Klage mit Gott direkt als Adressat lautet die allgemeine Ausgangsfrage für die Bearbeitung des Theodizeeproblems in den Worten des Psalmisten: »Gott, wie lange willst du mich noch leiden lassen? ${ }^{44}$ Varianten dieser Frage lauten: »Mein Gott, warum beziehungs-

40 Vgl. Augustinus, Bekenntnisse, Stuttgart 1998.

41 Was bedeutet die Infragestellung Gottes für die Möglichkeit, als sittliches Subjekt zu leben? Vgl. Gerd Neuhaus, Theodizee. Abbruch oder Anstoß des Glaubens, Freiburg u.a. 1993, $315 \mathrm{f}$.

42 Gotthard Fuchs, "Wir sind sein Kreuz«. Mystik und Theodizee, in: Ders., Angesichts des Leids an Gott glauben? Zur Theologie der Klage, Frankfurt 1996, 148-183, 172. Auch Oswald Bayer plädiert für eine Wiederentdeckung der Klage, insbesondere für eine Neuinterpretation aller dogmatischen Topoi vor dem Hintergrund der Kategorie der erhörten Klage. Vgl. Oswald BAyER, Zur Theologie der Klage, in: JBTh, Bd. 16 (2001), 289-301.

43 Nach Erika SCHUCHARDT blockiert argumentativer Trost häufig eher die Krisenverarbeitung. Vgl. Erika Schuchardt, Warum gerade ich...? Leiden und Glauben. Schritte mit Betroffenen und Begleitenden, Göttingen ${ }^{7} 1993$.

44 Vgl. Ps 13,2f. 
weise wozu hast du mich verlassen? « (Ps 22,2) und »Herr, wo bleibst du, wann kommst du endlich wieder? " Die Entscheidung für eine Ausgangsfrage in Gebetsform impliziert den Verzicht auf die Erwartung einer abstrakten Antwort, aber traut gleichzeitig Gott die Antwort zu, die der Mensch sich selbst nicht geben kann, nämlich die Antwort einer heilsamen Verwandlung. Bis dahin hält die Klage die notwendige Frage lebendig und offen. Die an Gott gerichtete Klage versucht nicht, Gott zu rechtfertigen, sondern behaftet ihn beim Leid seiner Schöpfung, nimmt seine Verantwortung für die Wirklichkeit in all ihren Aspekten ernst, ${ }^{45}$ aber kann gerade so auch auf seine Veränderungsmacht vertrauen und ihn bei seiner Verantwortung für die Überwindung des Übels behaften.

Die an Gott in der Du-Anrede gerichtete Frage setzt wenigstens einen Funken Vertrauen voraus und steht - auch wenn sie in der Form von Klage geäußert wird - in einer konstitutiven Beziehung zu vergangenem und zukünftigem Lob Gottes, wie es in vielen Klagepsalmen deutlich wird. Die Klage vor Gott stellt die Theodizeefrage an den für sie angemessenen Ort, nämlich die Gottesbeziehung. Denn schließlich haben wir es, wie schon Kant treffend formulierte, bei der Auseinandersetzung mit dem Theodizeeproblem weniger »mit einer Aufgabe zum Vorteil der Wissenschaft, als vielmehr mit einer Glaubenssache zu tun $«{ }^{46}$ Indem die Klage die VerschlieBung des Menschen in sich selbst durchbricht und Gott anspricht, ermöglicht sie Gottesbegegnung und neue Gottesoffenbarung. ${ }^{47}$ Der Gläubige, der Gott als abwesend erfährt, wirft dies dem abwesenden Gott in der (seine Anwesenheit voraussetzenden) Anrede vor. Metz fand für dieses Paradox die Formulierung "Gott um Gott bitten «, ${ }^{48}$ Luther nannte es "gegen Gott zu Gott fliehen «. ${ }^{49}$ Der siebente Vers des 42. Kapitels des Hiobbuches »denn ihr habt nicht recht von mir geredet wie mein Knecht Hiob « sollte nach Manfred Oeming besser übersetzt werden mit: »denn ihr habt nicht recht $z u$ mir geredet wie mein Knecht Hiob « ${ }^{50}$ Die Sprechrichtung, also der Adressat der Frage, ist entscheidend. Dass theologische Wissenschaft nicht $z u$, sondern über Gott redet, ist, so Oeming, nicht ihre Stärke, sondern ihre Schwäche. ${ }^{51}$

45 Gott schafft auch Unheil, vgl. Jes 6,9ff.; Jes 45,7; Ps 88,7ff.

46 Kant (s.o. Anm. 7), 194-225, 218.

47 Vgl. Sibylle Rolf, Crux sola est nostra theologia. Die Bedeutung der Kreuzestheologie für die Theodizeefrage, in: NZSTh 49 (2007), 223-240, 37 f.

48 Johann Baptist Metz, Memoria passionis. Ein provozierendes Gedächtnis in pluralistischer Gesellschaft, Freiburg 2006, 105.

49 WA $5,204,26 \mathrm{f}$.

50 Manfred Oeming/Konrad Schmid, Hiobs Weg. Stationen von Menschen im Leid, Neukirchen-Vluyn 2001, 138. Gott beantwortet Hiobs Frage »Gott, was habe ich getan, dass ich so leiden muss? « übrigens gerade nicht. Sondern indem Gottes Antwort lautet: »Wer bist du schon? «, korrigiert Gott Hiobs Fragestellung. Vgl. aaO., 116.

51 AaO., 140. 


\section{Fazit}

Welche Rolle spielt die Formulierung der Ausgangsfrage für die Bearbeitung des Theodizeeproblems? Die eingangs geäußerte Vermutung, dass von der Formulierung der Ausgangsfrage maßgeblich abhängt, $o b$ es eine Antwort gibt, welche Antwortmöglichkeiten in den Blick kommen können und welche nicht, welche Bedürfnisse des Fragenden befriedigt werden und welche nicht, kurz: was von der weiteren Problembearbeitung erwartet werden kann und was nicht, hat sich durch die Untersuchung - ohne dass ich bei den behandelten Aspekten einen Anspruch auf Vollständigkeit erhebe - bestätigt. Davon, ob die Ausgangsfrage im Horizont des Glaubens formuliert wird oder nicht, in existenzieller oder in abstrahierender Perspektive, mit Gott als Adressat oder nicht, anhand eines schöpfungstheologischen, kreuzestheologischen oder eschatologischen Zugangs, unter Zugrundelegung eines traditionell-theistischen oder eines nicht traditionell-theistischen Gottesbegriffs, agnostisch oder atheistisch, logisch oder empirisch, hängt es ab, wie viel Potenzial sie aus systematisch-theologischer Sicht enthält. Die Feststellung, dass allen diesen möglichen Frageformulierungen ein gemeinsamer Ausgangspunkt in der Spannung von Leidenserfahrung und Gottesglaube eignet, rechtfertigt nicht die Nivellierung ihrer tiefen Unterschiede. Vielmehr ist von jedem ernsthaften Beitrag zum Theodizeediskurs eine klare Benennung der jeweils zugrunde gelegten Ausgangsfrage und eine Begründung dieser Wahl zu fordern. Die einzig redliche Antwort auf Fragen, wie: »Ist die Theodizeefrage eine sinnvolle Frage? «, »Gibt es darauf eine Antwort? ", "Welches ist die überzeugendste Antwort? «, "Ist sie mit dem Glauben vereinbar? « usw., lautet: »Das kommt ganz auf die genaue Frage an." Der Ansatz bei der differenzierten Auseinandersetzung mit der Ausgangsfragestellung birgt meines Erachtens nicht nur ein Klärungspotenzial für den wissenschaftlichen Diskurs, sondern auch für die Gemeindearbeit und den Religionsunterricht ${ }^{52}$ und nicht zuletzt für das ganz persönliche Nachdenken.

52 Moderner Religionsunterricht betont weniger die Antwort des Lehrers, der vielleicht gar keine Frage des Schülers entspricht (Katechismustradition), sondern er möchte die »Fragebereitschaft des Kindes zur Geltung bringen und durch gemeinsames Fragen weiterentwickeln « im Sinne eines fragenorientierten religiösen Lernens. Rainer OвеRтнÜR, Kinder fragen nach Leid und Gott, München ${ }^{6} 2008,22$. Gegen Rainer Oberthür und gegen Karl Ernst Nipkow kommt die Untersuchung von Werner H. Ritter zu dem Ergebnis, dass das Theodizee-Problem (insbesondere bei abstrakten Formulierungen der Ausgangsfrage) für heutige Kinder und Jugendliche infolge eines kaum noch vorhandenen theistischen Gottesglaubens seine Brisanz verloren habe. Gleichwohl interessierten sich die Schüler nach wie vor für den Umgang mit Leid. Vgl. Werner H. Ritter/Helmut Hanisch/Erich Nestler/Christoph Gramzow, Leid und Gott. Aus der Perspektive von Kindern und Jugendlichen, Göttingen 2006. Hier stellt die Erschließung religiöser Formen der Klage eine vielversprechende Aufgabe dar. 


\section{ZUSAMMENFASSUNG}

Im Diskurs zum Theodizeeproblem beschäftigen sich die einzelnen Autoren mit sehr unterschiedlichen Ausgangsfragen, und zwar meist ohne diese Tatsache zu reflektieren. Wenn man jedoch ernst nimmt, dass »das Theodizeeproblem « einen Sammelbegriff für sehr unterschiedliche Fragen darstellt, setzt eine sinnvolle Bearbeitung des Theodizeeproblems eine bewusste Benennung der genauen Ausgangsfrage und idealerweise eine sachliche Begründung für die Wahl eben dieser Frage voraus. Davon, ob die Ausgangsfrage im Horizont des Glaubens formuliert wird oder nicht, in existenzieller oder in abstrahierender Perspektive, mit Gott als Adressat oder nicht, anhand eines schöpfungstheologischen, kreuzestheologischen oder eschatologischen Zugangs, unter Zugrundelegung eines traditionell-theistischen oder eines nicht traditionell-theistischen Gottesbegriffs, agnostisch oder atheistisch, logisch oder empirisch, hängt es ab, ob es eine Antwort gibt, welche Antwortmöglichkeiten in den Blick kommen können und welche nicht, welche Bedürfnisse des Fragenden befriedigt werden und welche nicht, kurz: was von der weiteren Problembearbeitung erwartet werden kann und was nicht.

\section{SUMMARY}

In the discourse on theodicy, individual authors deal with very different initial questions and for the most part fail to reflect upon this very fact. However, if one does recognize the importance of understanding THE problem of evil as an umbrella term for quite distinct issues, a proper treatment of theodicy implies precisely specifying the initial question and, ideally, a factual justification for the choice of this question. Whether the initial question is phrased in the horizon of faith, in an existential or abstract perspective, whether it directly adresses God, whether it is approached by means of creation theology, a theology of the cross or eschatological theology, whether it is based on a traditional theistical idea of god, agnostic or atheistic, logical or empirical, ultimately determines the actual existence of an answer, which possible answers can be brought to the questioner's attention and which of his or her needs can be satisfied in this regard; in short, what can be expected from the treatment of the problem and what cannot.

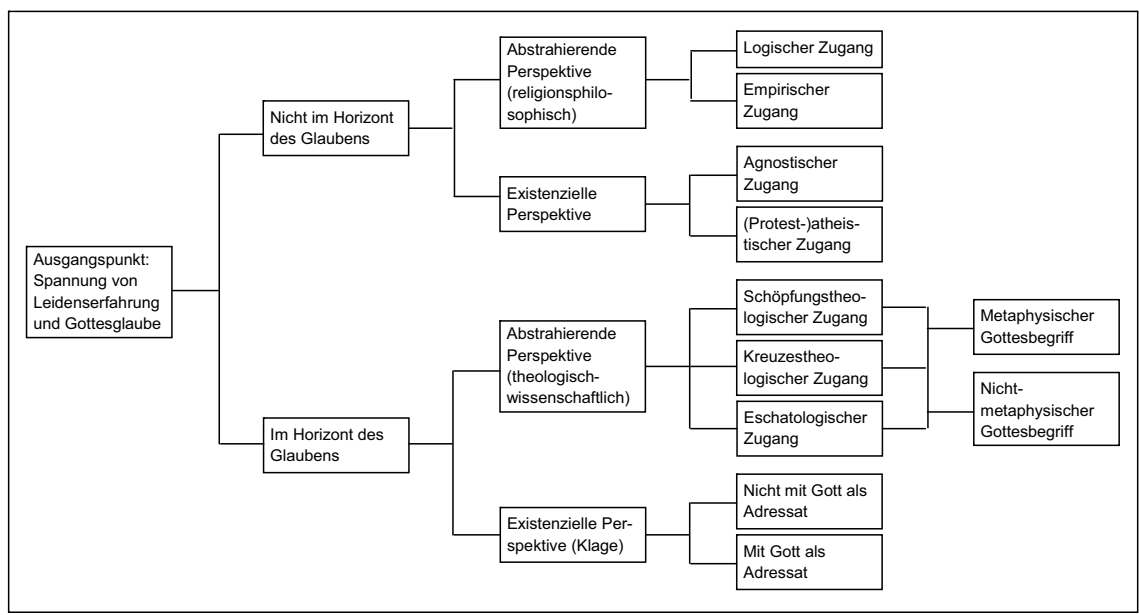

Abbildung 1: Kategorisierung möglicher Ausgangsfragen für die Bearbeitung des Theodizeeproblems 